\title{
Key Role for the Epsilon Isoform of Protein Kinase C in Painful Alcoholic Neuropathy in the Rat
}

\author{
Olayinka A. Dina, ${ }^{1}$ Justine Barletta, ${ }^{1}$ Xiaojie Chen, ${ }^{1}$ Annick Mutero, ${ }^{1}$ Annick Martin, ${ }^{2}$ Robert 0. Messing, ${ }^{2}$ and \\ Jon D. Levine ${ }^{1}$ \\ ${ }^{1}$ Departments of Medicine and Oral and Maxillofacial Surgery, Division of Neuroscience and Biomedical Sciences Program, \\ National Institutes of Health Pain Center (UCSF), University of California at San Francisco, San Francisco, California 94143- \\ 0440, and 2Department of Neurology, University of California at San Francisco and Ernest Gallo Clinic and Research \\ Center, Emeryville, California 94608
}

\begin{abstract}
Chronic alcohol consumption produces a painful peripheral neuropathy for which there is no reliably successful therapy, attributable to, in great part, a lack of understanding of the underlying mechanisms. We tested the hypothesis that neuropathic pain associated with chronic alcohol consumption is a result of abnormal peripheral nociceptor function. In rats maintained on a diet to simulate chronic alcohol consumption in humans, mechanical hyperalgesia was present by the fourth week and maximal at 10 weeks. Thermal hyperalgesia and mechanical allodynia were also present. Mechanical threshold of $\mathrm{C}$-fibers in ethanol fed rats was lowered, and the
\end{abstract}

number of action potentials during sustained stimulation increased. The hyperalgesia was acutely attenuated by intradermal injection of nonselective protein kinase $\mathrm{C}(\mathrm{PKC})$ or selective PKC $\epsilon$ inhibitors injected at the site of nociceptive testing. Western immunoblot analysis indicated a higher level of PKC $\epsilon$ in dorsal root ganglia from alcohol-fed rats, supporting a role for enhanced PKC $\epsilon$ secondmessenger signaling in nociceptors contributing to alcohol-induced hyperalgesia.

Key words: protein kinase $C \epsilon$; alcoholic peripheral neuropathy; pain; hyperalgesia; allodynia; primary afferent nociceptor
Ethanol consumption is the most common cause of peripheral nervous system, as well as CNS, neurotoxicity. Ethanol is thought to exert a direct neurotoxic action on the peripheral nervous system, resulting in a neuropathy that mostly involves smalldiameter fibers (Diamond and Messing, 1994; Monforte et al., 1995; Kielhorn, 1996; Ortiz-Plata et al., 1998; Tredici et al., 1999). The peripheral neuropathy is a potentially incapacitating complication of chronic consumption of ethanol, characterized by pain and dysesthesias, primarily in the lower extremities, and is poorly relieved by available therapies (Ratcliff, 1979; Monforte et al., 1995; Ortiz-Plata et al., 1998).

Whereas enhanced nociception and primary afferent nociceptor hypersensitivity have been demonstrated in animal models of other neuropathic pain states, such as those induced by diabetes (Ahlgren and Levine, 1994), chemotherapy (Tanner et al., 1998; Authier et al., 1999), or trauma (Bennett and Xie, 1988; Campbell et al., 1988; Seltzer et al., 1990; Xie and Xiao, 1990; Kim and Chung, 1992; Kim et al., 1993; Sheen and Chung, 1993; Yoon et al., 1996; Pedersen and Kehlet, 1998; Zahn and Brennan, 1999), an animal model for alcohol-induced neuropathy does not exist, nor has it even been demonstrated that primary afferent nociceptor function is altered by chronic exposure to alcohol.

In animal models of other painful peripheral neuropathies, enhanced nociception involves alterations in intracellular signaling. Specifically, protein kinase C (PKC) (Ahlgren and Levine, 1994) [particularly the epsilon $(\epsilon)$ isoform (Gerstin et al., 1998; Khasar et al., 1999] and protein kinase A (PKA) (Ahlgren and Levine, 1994) signaling pathways have been implicated in enhancing nociceptor function. Because alcohol has been shown to activate PKC and PKA (Coe et al., 1996; Pandey, 1996; Gerstin et al., 1998; Constantinescu et al., 1999; Sisson et al., 1999; Yoshimura and Tabakoff, 1999), we hypothesized that, in a well established model for

\footnotetext{
Received June 29, 2000; revised Aug. 24, 2000; accepted Aug. 30, 2000.

This work was funded by National Institutes of Health Grant DE08973 and funds provided by the state of California for medical research on alcohol and substance abuse through UCSF (R.O.M.).

Correspondence should be addressed to Dr. Jon D. Levine, National Institutes of Health Pain Center (UCSF), Box 0440, University of California at San Francisco, San Francisco, CA 94143-0440. E-mail: levine@itsa.ucsf.edu.

Copyright (C) 2000 Society for Neuroscience $0270-6474 / 00 / 208614-06 \$ 15.00 / 0$
}

chronic ethanol consumption in the rat, both enhanced nociception and nociceptor function occur and that PKC and PKA pathways contribute to the enhanced nociception.

\section{MATERIALS AND METHODS}

Chronic alcohol consumption. Male Sprague Dawley rats (200-450 gm; Bantin and Kingman, Fremont, CA), individually caged and maintained under a $12 \mathrm{hr}$ light/dark cycle, were used in these experiments. The experimental rats were fed Lieber-DeCarli liquid diet (Dyets Inc., Bethlehem, PA) (Lieber and DeCarli, 1982, 1989; Lieber et al., 1989) with ethanol (ED) (6.5\% ethanol) for 12 weeks. The control diet (CD) rats were pair-fed (i.e., calorically matched to the ethanol-exposed rats) by giving a diet in which equal calories of maltose-dextrin or alcohol (Gold Shield Chem Co., Hayward, CA) was consumed (Lieber et al., 1989). Using a subset of a group of rats that had been maintained for 12 weeks on ethanol diet, an alcohol withdrawal study was performed by switching the rats to $\mathrm{CD}$ for a period of 5 weeks.

The Institutional Animal Care Committee of the University of California, San Francisco, approved the experimental protocol.

Blood ethanol determination. Blood ethanol concentration was measured by drawing $100 \mu \mathrm{l}$ blood samples from the tail vein of ED rats into heparinized Eppendorf tubes. The blood samples, collected $3 \mathrm{hr}$ after commencement of feeding, were centrifuged to separate plasma. The samples were analyzed for alcohol by gas chromatography (Eriksson, 1973; Tabakoff et al., 1976). The blood samples were taken on the last day of the eighth week of ED to a separate group of rats, at which time behavioral testing had demonstrated a significant hyperalgesia.

Mechanical nociceptive threshold. The nociceptive flexion reflex was quantified using the Randall-Selitto paw pressure device (Stoelting, Chicago, IL), which applies a linearly increasing mechanical force to the dorsum of the rat's hindpaw (Taiwo et al., 1989b). The mechanical nociceptive threshold was defined as the force in grams at which the rat withdrew its paw. Rats were familiarized in the test apparatus at $5 \mathrm{~min}$ intervals for a period of $1 \mathrm{hr}$ per day for $3 \mathrm{~d}$ in the week preceding the experiment to decrease nociceptive thresholds (Taiwo et al., 1989b). The rat was allowed to crawl into a cotton sock, which is then placed on a Perspex block and covered with an elastic blanket that is attached to the block, on two sides, by Velcro strips. The rat was allowed to acclimatize in the restrainer as described for a period of $15-20 \mathrm{~min}$, after which it was exposed to the test stimulus (Taiwo et al., 1989b). Briefly, the training procedure consisted of repeated paw-withdrawal tests at 5 min intervals for $1 \mathrm{hr}$ per day. The stimulus was applied using a Basile analgesy meter, an instrument that applied a linearly increasing mechanical force onto the dorsum of the rat's paw at the same site at which test agents were to be injected. Baseline paw-withdrawal threshold was defined as the mean of the last six readings before test agents were injected. Behavioral testing was done on both $\mathrm{ED}$ and $\mathrm{CD}$ rats once per week, and the two groups were tested the same day. After 8 weeks of ethanol diet feeding, the decrease in 
paw-withdrawal threshold had plateaued, and test agents were injected intradermally into the dorsum of the hindpaws.

Stimulation with von Frey hair. Mechanical allodynia was assessed with von Frey hairs (VFH) using the up-down method, as described previously (Chaplan et al., 1994; Kinnman and Levine, 1995; Aley et al., 1996) Calibrated VFH $(1.32,3.63,10.0$, and $27.5 \mathrm{mN}$; Ainsworth, London, UK) were applied to the plantar skin of each hindpaw in these studies.

Thermal stimulation. The thermal nociceptive threshold was measured as described by Hargreaves et al. (Hargreaves et al., 1988), in both hindpaws, only once to avoid tissue injury (Aley et al., 1996).

In vivo electrophysiology. Single-fiber electrophysiological techniques were similar to those used in our previous studies of painful peripheral neuropathy (Tanner et al., 1998; Chen and Levine, 1999). Briefly, the rats were anesthetized with sodium pentobarbital (initially $50 \mathrm{mg} / \mathrm{kg}$, i.p., with additional doses given throughout the experiments to maintain areflexia). Single cutaneous C-fibers from the saphenous nerve were recorded. A tota of $10 \mathrm{C}$-fibers (one fiber per rat) were evaluated in this study. The action potential corresponding to the $\mathrm{C}$-fiber whose receptive field had been identified was determined by the latency delay technique, in which a mechanically induced orthodromic spike produced a delay in the electrically induced orthodromic spike (Iggo, 1958; Handwerker, 1991). The conduction velocity of a fiber was determined by dividing the distance between the stimulating and recording electrodes by the action potential latency. Fibers with conduction velocities $<2 \mathrm{~m} / \mathrm{sec}$ were classified as C-fibers (Willis, 1985)

Mechanical threshold of C-fibers was determined with calibrated (VFH) (Ainsworth, London, UK) and defined as the lowest force that elicited two or more spikes within $1 \mathrm{sec}$, in at least 6 of 10 trials. Sustained threshold stimulation was performed using a calibrated VFH that was placed, by hand, on the receptive field for $60 \mathrm{sec}$. Sustained $(60 \mathrm{sec})$ suprathreshold stimulation was performed with an apparatus consisting of a $10 \mathrm{gm}$ monofilament VFH connected in series with a mechanical force transducer (Entran, Fairfield, NJ). Three separate applications of the sustained stimulus were performed for each fiber; the average of the values for the three applications was taken as the response of that fiber.

Western blot analysis for PKC $\epsilon$. Eight lumbar dorsal root ganglia (DRGs) $\left(\mathrm{L}_{2}-\mathrm{L}_{5}\right.$ bilaterally) were harvested from both ethanol-treated and control rats. The DRGs were desheathed, frozen in a dry ice ethanol bath, and stored at $-80^{\circ} \mathrm{C}$. The samples were homogenized in 5 vol of $20 \mathrm{~mm}$ Tris-HCl, pH 7.5, 2 mM EDTA, 10 mM EGTA, 1 mM phenylmethylsulphonyl fluoride, and $20 \mu \mathrm{g} / \mathrm{ml}$ each of leupeptin, aprotinin, and pepstatin using a pestle (Pellet Pestle Motor; Kontes, Vineland, NJ). After homogenization, the samples were spun at $13,000 \mathrm{rpm}$ for $15 \mathrm{~min}\left(4^{\circ} \mathrm{C}\right)$. The supernatants were collected, and a portion of each was used for protein quantification (Micro BCA Protein Assay Reagent kit; Pierce, Rockford, IL). Concentrated sample buffer was added to each sample so that the final solutions contained $56 \mathrm{~mm}$ Tris- $\mathrm{HCl}, 5 \%$ glycerol, $1.67 \%$ SDS, and 0.02 $\mathrm{mg} / \mathrm{ml}$ bromophenol blue. The samples were then boiled for $10 \mathrm{~min}$, cooled on ice, and stored at $4^{\circ} \mathrm{C}$. Protein samples $(8.3 \mu \mathrm{g} /$ lane $)$ were separated by SDS-PAGE on $8 \%$ minigels and thereafter transferred to polyvinylidene difluoride (PVDF) transfer membranes (Immobilon-P; Millipore, Bedford, MA) for $1 \mathrm{hr}$ at $100 \mathrm{~V}$. The membranes were washed briefly with TBS (20 $\mathrm{mm}$ Tris- $\mathrm{HCl}, \mathrm{pH} \mathrm{7.6}$, and $150 \mathrm{~mm} \mathrm{NaCl}$ ) and then blocked for $1 \mathrm{hr}$ in Blotto [5\% nonfat dry milk in TBS containing $0.05 \%$ Tween 20 (TBS-T)]. After blocking, the blots were incubated at room temperature for $45 \mathrm{~min}$ in Blotto with $1 \mu \mathrm{g} / \mathrm{ml}$ rabbit polyclonal antibody to PKC $\epsilon$ (Santa Cruz Biotechnology, Santa Cruz, CA) and $0.05 \%$ sodium azide. Blots were washed three times for $10 \mathrm{~min}$ with TBS-T, incubated at room temperature for $30 \mathrm{~min}$ in Blotto with HRP-conjugated goat anti-rabbit IgG antibody at 1:4000 dilution (Vector Laboratories, Burlingame, CA), and then washed again three times for $12 \mathrm{~min}$ in TBS-T and once in TBS alone. PKC $\epsilon$ bands were visualized using the ECL ${ }^{\text {lus }}$ kit (Amersham Pharmacia Biotech, Little Charlton, Buckinghamshire, UK). Autoradiograms of the blots were then digitized, and the density of the immunoreactive bands was quantified, in arbitrary units, using NIH Image software.

Drugs. Bisindolylmaleimide (BIMM) (nonspecific PKC inhibitor), $\mathrm{PKC} \epsilon$ specific translocation inhibitor peptide (PKC $\epsilon-\mathrm{I})$ (Johnson et al., 1996; Khasar et al., 1999) (Calbiochem, La Jolla, CA), and Walsh inhibitor peptide (WIPTIDE) (PKA inhibitor; Peninsula Laboratories, Belmont, CA) were used. Stock solutions $(1 \mu \mathrm{g} / \mu \mathrm{l})$ of BIMM (in $10 \%$ dimethylsulfoxide) and PKC $\epsilon$-I and WIPTIDE (in $0.9 \%$ saline) were stored at $-20^{\circ} \mathrm{C}$. All inhibitors were diluted with distilled water before intradermal injections into the paw. The effects of the various antagonists, BIMM, PKC $\epsilon-\mathrm{I}$, and WIPTIDE, were assessed to determine second-messenger system(s) contributing to alcohol-induced hyperalgesia. Injections of PKC $\epsilon-\mathrm{I}$ and WIPTIDE $(1 \mu \mathrm{g} / 2.5 \mu \mathrm{l})$, using a $10 \mu \mathrm{l}$ microsyringe (Hamilton, Reno, $\mathrm{NV}$ ), were always preceded by injection of distilled water $(2.5 \mu \mathrm{l})$ to produce hypo-osmotic shock, thereby enhancing cell membrane permeability to these cell membrane-impermeable agents (Tsapis and Kepes, 1977; West and Huang, 1980; Taiwo and Levine, 1989; Khasar et al., 1995; Widdicombe et al., 1996). The dose of each protein kinase inhibitor was separated from the distilled water by an air bubble $(<1 \mu l)$. Pawwithdrawal threshold was remeasured 10,15 , and 20 min after injecting the test agent. The mean of the paw-withdrawal thresholds obtained at these three points was then taken as the mechanical nociceptive threshold at the dose of the test agent used. The effect of each dose of a test agent was calculated as the percentage change from baseline.

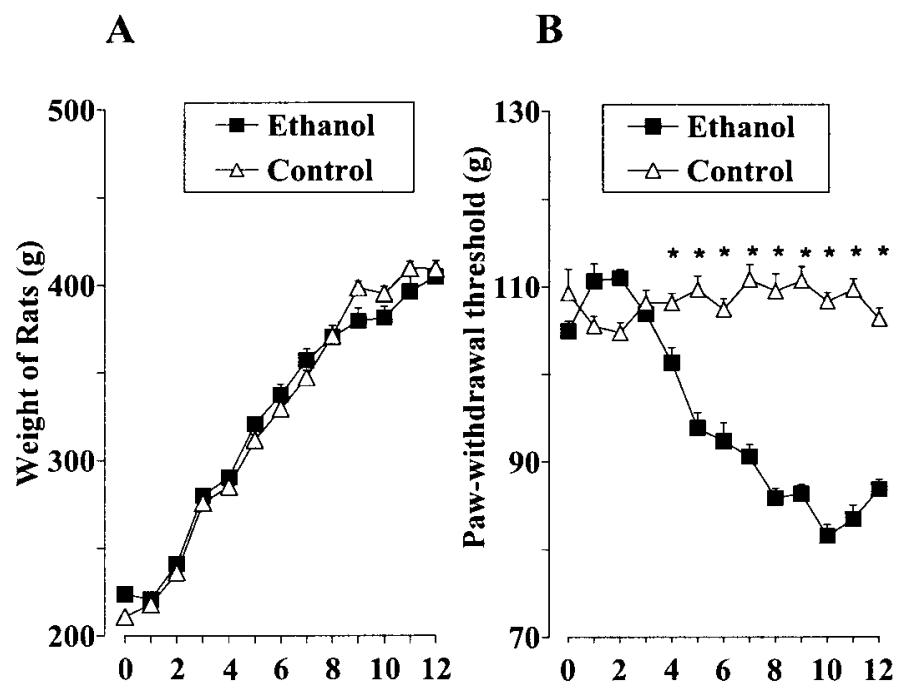

Time (Weeks)

Figure 1. A, Effect of long-term ethanol feeding on body weight using the Lieber-DeCarli liquid diet pair-feeding technique. The average weight of ethanol-fed ( filled squares; $n=17$ ) and isocalorically fed control (open triangles; $n=12$ ) rats is plotted against the duration of administration of the liquid diet. Each point represents mean \pm SEM weight (in grams) (error bars). $B$, Decrease in paw-withdrawal threshold to mechanical stimulation of the dorsum of the rat hindpaw induced by chronic administration of ethanol (alcohol-induced mechanical hyperalgesia). The paw-withdrawal threshold (in grams) of ethanol-fed ( filled squares; $n=34$ paws) and control diet-fed (open triangles; $n=24$ paws) rats plotted against the duration (in weeks) of chronic exposure to ethanol administered by the Lieber-DeCarli liquid diet technique and pair-fed isocaloric diet controls. From the fourth week of ethanol diet feeding, ${ }^{*} p<0.0001$ (repeated measures ANOVA).

Statistical analysis. The data are presented as mean \pm SEM and were compared using the Student's $t$ test, ANOVA [one-way or repeated measures followed by Fisher's protected least significant difference (PLSD) post hoc analysis] or Mann-Whitney $U$ test, as appropriate. $p \leq 0.05$ was considered statistically significant.

\section{RESULTS}

\section{Effect on weight of chronic exposure to ethanol compared with an isocaloric diet}

Just before initiation of the Lieber-DeCarli liquid diets, rats assigned to the CD group weighed $211.0 \pm 1.0 \mathrm{gm}$ compared with $223.8 \pm 1.7 \mathrm{gm}$ in rats assigned to the ED group (Fig. $1 A$ ). Animals in the two groups gained weight comparably throughout the period of study. After 12 weeks of feeding with the Lieber-DeCarli diets, $\mathrm{CD}$ and ED rats weighed $409.3 \pm 4.7$ and $405.0 \pm 9.3 \mathrm{gm}$, respectively $(p>0.05)$ (Fig. $1 A)$. Blood ethanol concentration was determined as $66.8 \pm 7.0 \mathrm{mmol} / 1$ (mean \pm SEM; range, 46.4-92.6 $\mathrm{mmol} / \mathrm{l} ; n=6$ ethanol-treated rats).

\section{Effect of ethanol on mechanical nociceptive threshold (mechanical hyperalgesia)}

Before the administration of the liquid diets, the mean baseline paw-withdrawal threshold of ED rats $(104.9 \pm 1.1 \mathrm{gm} ; n=34)$ (Fig. $1 B)$ was not significantly different $(p>0.05)$ from that in CD rats $(109.3 \pm 2.8 \mathrm{gm} ; n=24)$. A significant decrease in mechanical nociceptive threshold (i.e., hyperalgesia) was produced in the ED rats after 4 weeks $(p<0.0001 ; F=234.1)$ (Fig. $1 B)$. Further reduction $(p<0.05)$ in the paw-withdrawal threshold of ED rats to $81.6 \pm 1.3 \mathrm{gm}$ occurred at 10 weeks, after which hyperalgesia was maintained in the range of 81.6-84.1 gm until the conclusion of the study, at 12 weeks. There was no significant change $(p>0.05)$ in the mean paw-withdrawal threshold of CD rats over the same time period. 
A

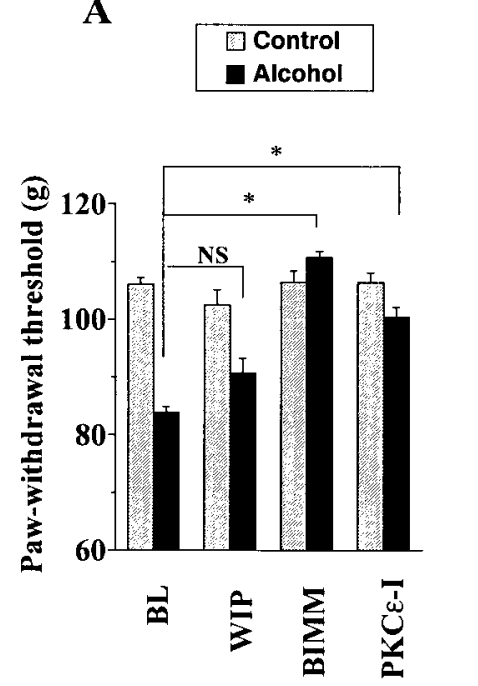

B

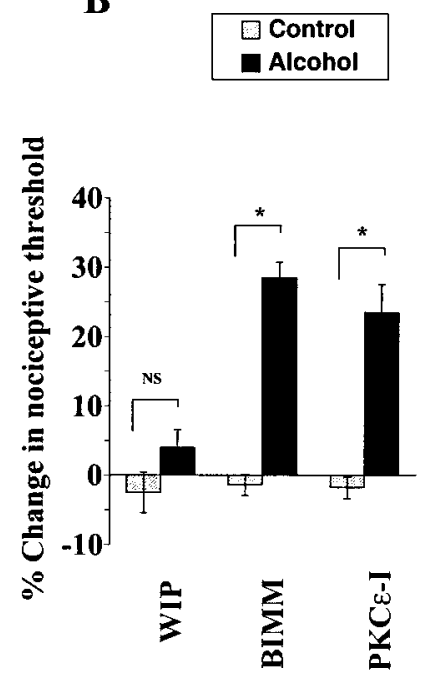

Figure 2. $A$, Effect of WIPTIDE (WIP; $1 \mu \mathrm{g} ; n=12$ paws), BIMM $(1 \mu \mathrm{g}$; $n=12$ paws), and PKC $\epsilon$-I ( $1 \mu \mathrm{g} ; n=12$ paws) on the baseline $(B L)$ paw threshold (grams \pm SEM) of ethanol-fed (filled bars; $n=12$ paws) and isocalorically fed control (hatched bars; $n=12$ paws) rats. ${ }^{*} p<0.0001$ (ANOVA, followed by Fisher's PLSD post hoc test). NS, Not statistically significant. $B$, Reduction in lowered paw-withdrawal threshold (hyperalgesia) produced by WIPTIDE (WIP; $1 \mu \mathrm{g} ; n=12$ paws), BIMM (1 $\mu \mathrm{g} ; n=$ 12 paws), and PKC $\epsilon$-I ( $1 \mu \mathrm{g} ; n=12$ paws $)$ plotted as percentage change in nociceptive threshold in ethanol-fed (filled bars; $n=12$ paws) and isocalorically fed control (hatched bars; $n=12$ paws) rats. * $p<0.0001$ (one-way ANOVA and Fisher's PLSD post hoc test). NS, Not statistically significant.

\section{Role of PKC/PKC $\epsilon$ and PKA in ethanol-induced mechanical hyperalgesia}

After 8 weeks of ethanol administration, the intradermal injection of BIMM or PKC $\epsilon$-I (each administered at $1 \mu \mathrm{g}$ ) resulted in a significant reduction in mechanical hyperalgesia in ED rats (each $p<0.0001 ; F=14.8$ ) (Fig. $2 A, B$ ). There was no significant difference $(p>0.05)$ in the effect of these two inhibitors on paw-withdrawal threshold in the hyperalgesic ED rats. WIPTIDE did not significantly $(p=0.07)$ affect nociceptive threshold in hyperalgesic ED or nonhyperalgesic $\mathrm{CD}$ rats, although there was a trend toward an effect.

\section{von Frey hair stimulation (mechanical allodynia)}

To determine whether the mechanical hyperalgesia induced by alcohol was associated with mechanical allodynia, we examined responses to von Frey hair stimulation in ED and CD rats. ED rats demonstrated a significantly $(p<0.05)$ increased response frequency to $27.5 \mathrm{mN}$ VFH stimulation (Fig. $3 A$ ) when compared with the response in $\mathrm{CD}$ rats.

\section{Thermal hyperalgesia}

Because thermal hyperalgesia also occurs in patients with neuropathic pain, we compared thermal responses in $\mathrm{ED}$ and $\mathrm{CD}$ rats using the method of Hargreaves et al. (1988). ED rats demonstrated a significantly $(p<0.05)$ lower $(6.8 \pm 0.4 \mathrm{sec})$ pawwithdrawal latency in response to thermal stimulation when compared with $C D$ rats $(8.6 \pm 0.5 \mathrm{sec})$ (Fig. $3 B)$. Thus, ethanol treatment evoked thermal hyperalgesia.

\section{Effect of ethanol withdrawal on ethanol-induced hyperalgesia}

After 12 weeks of ethanol diet, a subset, comprised of three rats, from the ED rats were placed on the control diet to determine whether ethanol-induced hyperalgesia was reversible. In ED rats placed on a $\mathrm{CD}$ for 2 weeks, the paw-withdrawal threshold was not significantly different $(p>0.05)$ than it had been after 12 weeks on the ethanol diet (Fig. 4). Interestingly, 5 weeks after being placed on the $\mathrm{CD}$, the nociceptive threshold in this group was even more reduced than during the last week of ethanol treatment $(p<$

A

B

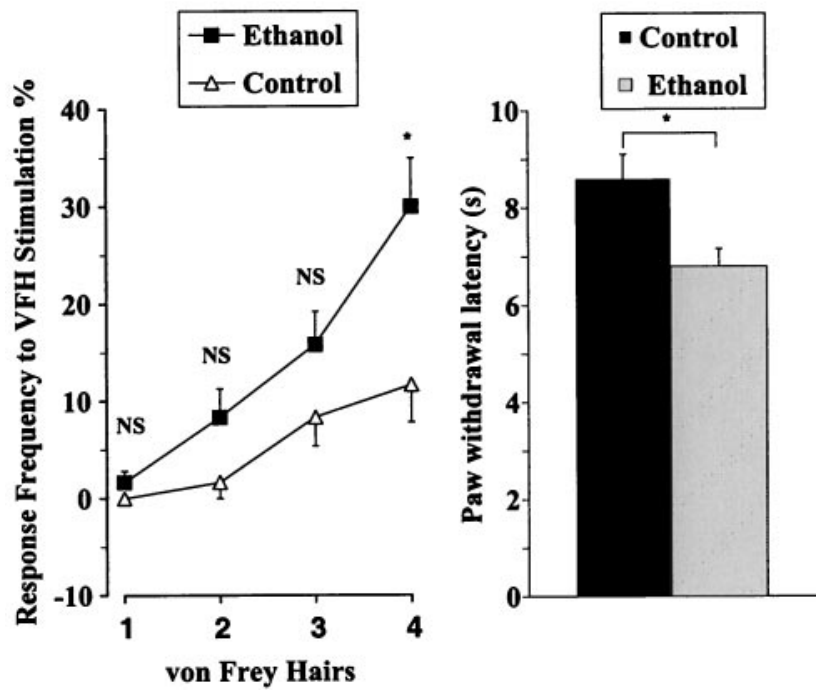

Figure 3. A, Effect of chronic alcohol consumption on response to stimulation by calibrated $1.32,3.63,10.0$, and $27.5 \mathrm{mN}$ (represented on the abscissa by $1,2,3$, and 4 ) VFH measured in ethanol-fed ( filled squares; $n=$ 24 paws) and control-diet fed (open triangles; $n=12$ paws) rats. ${ }^{*} p<0.05$ (repeated measures ANOVA). NS, Not statistically significant. $B$, Effect on sensitivity, as measured by the paw-withdrawal latency, to thermal stimulation from a $50 \mathrm{~W}$ radiant heat stimulus, in ethanol-fed ( filled bar; $n=12$ paws) and control (hatched bar; $n=12$ paws) rats. ${ }^{*} p<0.03$ (Student's unpaired $t$ test).

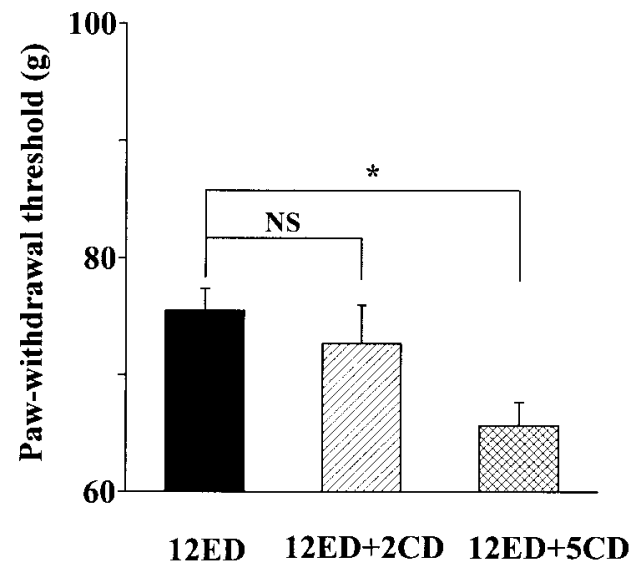

Time (Weeks)

Figure 4. Effect of ethanol withdrawal (initiation of the CD in a group of rats fed ED in the preceding 12 weeks) on the paw threshold (in grams) of rats fed for 12 weeks on ED (12ED, filled bar; $n=34$ paws) and at 2 $(12 E D+2 C D$, hatched bar; $n=6$ paws $)$ and $5(12 E D+5 C D$, cross-hatched bar; $n=6$ paws) weeks after initiation of CD. * $p<0.0002$. NS, Not significant $(p>0.05)$. Comparisons were by one-way ANOVA and Fisher's post hoc test.

0.0002), indicating that hyperalgesia had increased during the 5 week period on CD (Fig. 4).

\section{Effect of ethanol on C-fiber threshold and responsivity}

Study of C-fibers from ED and CD rats revealed no spontaneous activity in most fibers. In a few fibers, spontaneous activity, although present, was $<0.01 \mathrm{~Hz}$. The conduction velocities for C-fibers in ED and CD rats were $0.9 \pm 0.1$ and $0.8 \pm 0.1 \mathrm{~m} / \mathrm{sec}$, respectively $(p>0.05)$ (Fig. $5 A)$. The location of receptive fields of $\mathrm{C}$-fibers on the dorsum of the hindpaw from $\mathrm{CD}$ and $\mathrm{ED}$ rats was also similar. However, the mean mechanical threshold for C-fibers from the ED rats was lower than that from $\mathrm{CD}$ rats $(p<0.05)$ (Fig. 
A
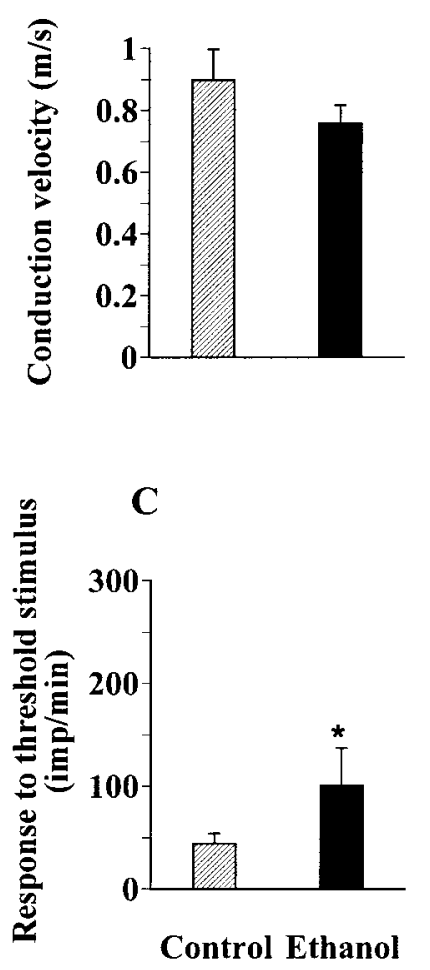

Figure 5. Conduction velocity and mechanical threshold of C-fibers in CD and ED rats. $A$, There was no difference in conduction velocity between C-fibers from ED ( filled bar; $n=10$ ) and CD (hatched bar; $n=14$ ) rats. Unpaired $t$ test, $p>0.05$. $B$, The mechanical threshold of $C$-fibers from ED rats ( filled bar; $n=10$ ) was significantly lower than that of C-fibers from control rats (hatched bar; $n=14$ ) using nonparametric Mann-Whitney $U$ test; ${ }^{*} p<0.05$. $C$, The number of action potentials evoked by sustained $(60$ sec) threshold stimulation was significantly greater in C-fibers in ED rats ( filled bar; $n=10$ ) compared with controls (hatched bar; $n=14$ ). $D$, The number of action potentials evoked by sustained suprathreshold (10 gm) stimulation was significantly greater in C-fibers in ethanol-fed rats (filled bar; $n=10$ ) compared with controls (hatched bar; $n=14$ ). Unpaired (one-tailed) $t$ test; ${ }^{*} p<0.05$ (Student's unpaired $t$ test).

$5 B)$. Also, the mean number of action potentials evoked by a sustained $60 \mathrm{sec}$ threshold or suprathreshold (10 gm) stimulus was significantly higher for $\mathrm{C}$-fibers from $\mathrm{ED}$ rats compared with those from $\mathrm{CD}$ rats $(p<0.05)$ (Fig. $5 C, D)$.

\section{PKC $\epsilon$ level}

To determine whether the PKC $\epsilon$-dependent decrease in nociceptive threshold observed in ED rats is associated with a higher level of expression of PKC $\epsilon$ in primary afferents, a Western analysis of DRG protein samples from $C D$ and ED rats was performed. There was a higher level of PKC $\epsilon$ in dorsal root ganglia in ED rats (Fig. $6)$. When the PKC $\epsilon$-immunoreactive bands observed were quantified, the average PKC $\epsilon$ level in alcohol-fed $(n=11)$ rats was found to be $50 \%$ higher $(p<0.001)$ (Fig. $6 A, B)$ than that in the control rats $(n=10)$.

\section{DISCUSSION}

We have developed the first animal model for alcohol-induced painful peripheral neuropathy and describe alterations in primary afferent nociceptor function and in specific second-messenger signaling that contributes to the enhanced nociception. Rats chronically fed ethanol exhibited mechanical and thermal hyperalgesia and tactile allodynia, all of which are symptoms frequently occurring in patients with painful peripheral neuropathy (Scadding, 1992). This model is highly relevant to painful alcoholic neuropathy in humans because the blood alcohol level that results in neuropathic changes is similar (Bosch et al., 1979; Lieber and
$\mathbf{A}$
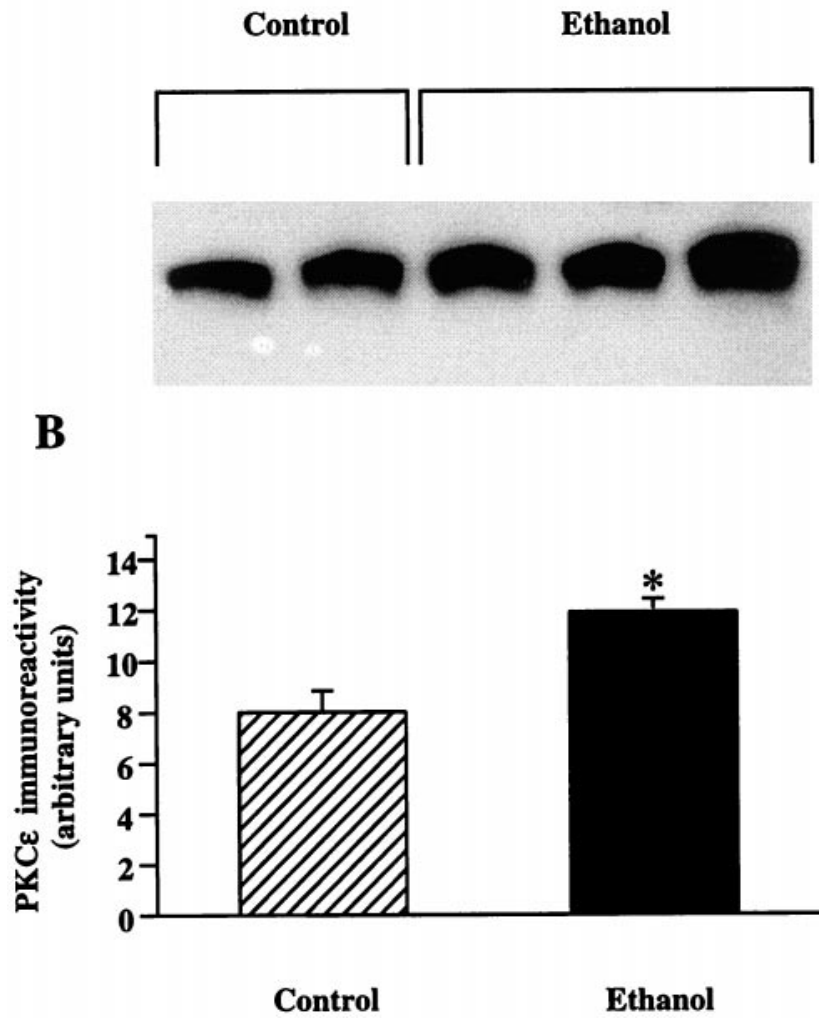

Figure 6. PKC $\epsilon$ levels in DRG from $\mathrm{CD}$ and $\mathrm{ED}$ rats. $A$, Representative blot of PKC immunoreactivity in DRG samples from CD and ED rats (after 10 weeks of ethanol administration). Proteins were separated by SDSPAGE, transferred to a PVDF membrane, and immunoblotted with PKC $\epsilon$ specific antibody. $B$, Mean \pm SEM for data showed a statistically significant difference between control diet-fed (hatched bar; $n=10$ ) and ethanol-fed ( filled bar; $n=11$ ) rats. ${ }^{*} p<0.01$ (Student's $t$ test).

DeCarli, 1989; Lieber et al., 1989). Also, the fact that hyperalgesia is stable for weeks renders this model very useful for study of underlying mechanism.

Ethanol-induced hyperalgesia did not resolve and, in fact, continued to increase at 5 weeks after cessation of alcohol intake. This is consistent with reports of persisting or even worsening peripheral neuropathy in patients who have withdrawn from chronic ethanol use (Gadner, 1972; Weise et al., 1985; Yokoyama et al., 1991; Spahn et al., 1995).

We tested whether two intracellular second-messenger pathways contribute to ethanol-induced hyperalgesia. PKC and PKA are both known to be important in nociceptor function (Taiwo et al., 1989a; Ahlgren and Levine, 1994; Khasar et al., 1999) and in mediating other effects of alcohol (Diamond et al., 1987; Hoffman et al., 1987; Hoek et al., 1988, 1992; Messing et al., 1991; Lovinger and Zhou, 1994; Gordon et al., 1997). PKC was found to contribute to the enhanced nociception, whereas cAMP/PKA, if it does contribute, appears to be less important. This second-messenger dependence differs from that for the enhanced nociception produced by hyperalgesic inflammatory mediators, to which both PKA and PKC contribute (Taiwo et al., 1989a; Taiwo and Levine, 1991; Khasar et al., 1999). Because the PKC $\epsilon$ antagonist (PKC $\epsilon-\mathrm{I})$ alone was able to reverse the hyperalgesia to the same extent as the nonspecific PKC antagonist BIMM, the epsilon isoform of PKC accounts for the role of PKC in ethanol-induced enhanced nociception (Khasar et al., 1999). Because the PKC $\epsilon$ antagonist was able to attenuate established ethanol-induced hyperalgesia, ongoing $\mathrm{PKC} \epsilon$ activity must be required to maintain the hyperalgesia. A role for the $\epsilon$ isoform of PKC is consistent with previous demonstrations that chronic ethanol exposure increases levels of PKC $\epsilon$ in 
cultured cells (Messing et al., 1991; Coe et al., 1996) and that ethanol consumption causes sustained translocation (activation) of PKC $\epsilon$, but not of PKC $\delta$ or PKC $\alpha$, in cardiac tissue (Miyamae et al., 1999). PKC activity also contributes to neuropathic pain induced by cancer chemotherapy (vincristine) (K. O. Aley and J. D. Levine, unpublished observation) and diabetes (Ahlgren and Levine, 1994). The fact that neither PKC antagonist had an effect on mechanical threshold in control animals strongly supports the suggestion that the contribution of PKC $\epsilon$ to enhanced nociception develops during chronic ethanol exposure.

It appears, for several reasons, that the PKC $\epsilon$ activity that maintains ethanol-induced hyperalgesia occurs in the peripheral nociceptor terminal. First, we observed hyper-responsiveness (i.e., decrease in threshold and enhanced responsivity to mechanical stimuli) in C-fiber nociceptors. Second, intradermal injections of extremely small amounts of inhibitors attenuated the enhanced nociception. Finally, at the site of study, the skin of the hindpaw, PKC $\epsilon$ is believed to be expressed only in primary afferent terminals (Khasar et al., 1999).

In summary, we have demonstrated that hyperalgesia is present in an established model in the rat for chronic alcohol consumption in humans and that PKC $\epsilon$ signaling plays a critical role in the enhanced nociception produced by chronic alcohol. The findings suggest that PKC $\epsilon$ might be an excellent therapeutic target for this common and, at present, primarily untreatable chronic pain syndrome.

\section{REFERENCES}

Ahlgren SC, Levine JD (1994) Protein kinase C inhibitors decrease hyperalgesia and $\mathrm{C}$-fiber hyperexcitability in the streptozotocin-diabetic rat. J Neurophysiol 72:684-692.

Aley KO, Reichling DB, Levine JD (1996) Vincristine hyperalgesia in the rat: a model of painful vincristine neuropathy in humans. Neuroscience 73:259-265.

Authier N, Coudore F, Eschalier A, Fialip J (1999) Pain related behaviour during vincristine-induced neuropathy in rats. NeuroReport 10:965-968

Bennett GJ, Xie YK (1988) A peripheral mononeuropathy in rat that produces disorders of pain sensation like those seen in man. Pain 33:87-107.

Bosch EP, Pelham RW, Rasool CG, Chatterjee A, Lash RW, Brown L, Munsat TL, Bradley WG (1979) Animal models of alcoholic neuropathy: morphologic, electrophysiologic, and biochemical findings. Muscle Nerve 2:133-144.

Campbell JN, Raja SN, Meyer RA, Mackinnon SE (1988) Myelinated afferents signal the hyperalgesia associated with nerve injury. Pain 32:89-94.

Chaplan SR, Bach FW, Pogrel JW, Chung JM, Yaksh TL (1994) Quantitative assessment of tactile allodynia in the rat paw. J Neurosci Methods 53:55-63.

Chen X, Levine JD (1999) NOS inhibitor antagonism of PGE2-induced mechanical sensitization of cutaneous $\mathrm{C}$-fiber nociceptors in the rat. J Neurophysiol 81:963-966.

Coe IR, Yao L, Diamond I, Gordon AS (1996) The role of protein kinase C in cellular tolerance to ethanol. J Biol Chem 271:29468-29472.

Constantinescu A, Diamond I, Gordon AS (1999) Ethanol-induced translocation of CAMP-dependent protein kinase to the nucleus. Mechanism and functional consequences. J Biol Chem 274:26985-26991.

Diamond I, Messing RO (1994) Neurologic effects of alcoholism. West J Med 161:279-287.

Diamond I, Wrubel B, Estrin W, Gordon A (1987) Basal and adenosine receptor-stimulated levels of cAMP are reduced in lymphocytes from alcoholic patients. Proc Natl Acad Sci USA 84:1413-1416.

Eriksson CJ (1973) Ethanol and acetaldehyde metabolism in rat strains genetically selected for their ethanol preference. Biochem Pharmacol 22:2283-2292.

Gadner AJ (1972) Neuropathy after alcohol withdrawal. J Neurol Neurosurg Psychiatry 33:323-325.

Gerstin Jr EH, McMahon T, Dadgar J, Messing RO (1998) Protein kinase Cdelta mediates ethanol-induced up-regulation of L-type calcium channels. J Biol Chem 273:16409-16414.

Gordon AS, Yao L, Wu ZL, Coe IR, Diamond I (1997) Ethanol alters the subcellular localization of delta and epsilon protein kinase C NG 105-15 cells. Mol Pharmacol 52:554-559.

Handwerker HO (1991) Electrophysiological mechanisms in inflammatory pain. Agents Actions Suppl 32:91-99.

Hargreaves K, Dubner R, Brown F, Flores C, Joris J (1988) A new and sensitive method for measuring thermal nociception in cutaneous hyperalgesia. Pain 32:77-88.

Hoek JB, Rubin R, Thomas AP (1988) Ethanol-induced phospholipase C activation is inhibited by phorbol esters in isolated hepatocytes. Biochem J 251:865-871.
Hoek JB, Thomas AP, Rooney TA, Higashi K, Rubin E (1992) Ethanol and signal transduction in the liver. FASEB J 6:2386-2396.

Hoffman PL, Valverius P, Kwast M, Tabakoff B (1987) Comparison of the effects of ethanol on beta-adrenergic receptors in heart and brain. Alcohol Alcohol [Suppl] 1:749-754.

Iggo A (1958) The electrophysiological identification of single nerve fibers, with particular reference to the slowest-conduction vagal afferent fibers in the cat. J Physiol (Lond) 142:110-126.

Johnson JA, Gray MO, Chen CH, Mochly-Rosen D (1996) A protein kinase $\mathrm{C}$ translocation inhibitor as an isozyme-selective antagonist of cardiac function. J Biol Chem 271:24962-24966.

Khasar SG, Wang JF, Taiwo YO, Heller PH, Green PG, Levine JD (1995) $\mathrm{Mu}$-opioid agonist enhancement of prostaglandin-induced hyperalgesia in the rat: a G-protein beta gamma subunit-mediated effect? Neuroscience 67:189-195.

Khasar SG, Lin YH, Martin A, Dadgar J, McMahon T, Wang D, Hundle B, Aley KO, Isenberg W, McCarter G, Green PG, Hodge CW, Levine JD, Messing RO (1999) A novel nociceptor signaling pathway revealed in protein kinase C epsilon mutant mice. Neuron 24:253-260.

Kielhorn FW (1996) The history of alcoholism: Bruhl-Cramer's concepts and observations. Addiction 91:121-128.

Kim SH, Chung JM (1992) An experimental model for peripheral neuropathy produced by segmental spinal nerve ligation in the rat. Pain 50:355-363.

Kim SH, Na HS, Sheen K, Chung JM (1993) Effects of sympathectomy on a rat model of peripheral neuropathy. Pain 55:85-92.

Kinnman E, Levine JD (1995) Involvement of the sympathetic postganglionic neuron in capsaicin-induced secondary hyperalgesia in the rat. Neuroscience 65:283-291.

Lieber CS, DeCarli LM (1982) The feeding of alcohol in liquid diets: two decades of applications and 1982 update. Alcohol Clin Exp Res 6:523-531.

Lieber CS, DeCarli LM (1989) Liquid diet technique of ethanol administration: 1989 update. Alcohol Alcohol 24:197-211.

Lieber CS, DeCarli LM, Sorrell MF (1989) Experimental methods of ethanol administration. Hepatology 10:501-510.

Lovinger DM, Zhou Q (1994) Alcohols potentiate ion current mediated by recombinant 5-HT3RA receptors expressed in a mammalian cell line. Neuropharmacology 33:1567-1572.

Messing RO, Petersen PJ, Henrich CJ (1991) Chronic ethanol exposure increases levels of protein kinase $\mathrm{C}$ delta and epsilon and protein kinase C-mediated phosphorylation in cultured neural cells. J Biol Chem 266:23428-23432.

Miyamae M, Rodriguez MM, Camacho SA, Diamond I, Mochly-Rosen D, Figueredo VM (1999) Activation of epsilon protein kinase C correlates with a cardioprotective effect of regular ethanol consumption. Proc Natl Acad Sci USA 95:9262-8267.

Monforte R, Estruch R, Valls-Sole J, Nicolas J, Villalta J, Urbano-Marquez A (1995) Autonomic and peripheral neuropathies in patients with chronic alcoholism. A dose-related toxic effect of alcohol. Arch Neurol 52:45-51.

Ortiz-Plata A, Palencia G, Garcia E, Perez R, Sotelo J (1998) Ultrastructural changes in limb distal nerves of rats with alcoholism and/or malnutrition before and after dietary correction. J Appl Toxicol 18:89-92.

Pandey SC (1996) Protein kinase C: molecular and cellular targets for the action of ethanol. Alcohol Clin Exp Res 20:67A-71A.

Pedersen JL, Kehlet H (1998) Secondary hyperalgesia to heat stimuli after burn injury in man. Pain 76:377-384.

Ratcliff EV (1979) Alcoholic neuropathies. Aust Fam Physician 8:171-177.

Scadding JW (1992) Neuropathic pain. In: Diseases of the nervous system. Clinical neurobiology, Vol 2 (Asbury AK, McKhann GM, McDonald WI, eds), pp 858-872. Philadelphia: Saunders.

Seltzer Z, Dubner R, Shir Y (1990) A novel behavioral model of neuropathic pain disorders produced in rats by partial sciatic nerve injury. Pain 43:205-218.

Sheen K, Chung JM (1993) Signs of neuropathic pain depend on signals from injured nerve fibers in a rat model. Brain Res 610:62-68.

Sisson JH, May K, Wyatt TA (1999) Nitric oxide-dependent ethanol stimulation of ciliary motility is linked to cAMP-dependent protein kinase (PKA) activation in bovine bronchial epithelium. Alcohol Clin Exp Res 23:1528-1533.

Spahn TW, Lohse AW, Otto G, Tettenborn B, Hopf HC, Meyer zum Buschenfelde KH (1995) Remission of severe alcoholic polyneuropathy after liver transplantation. Z Gastroenterol 33:711-714.

Tabakoff B, Anderson RA, Ritzmann RF (1976) Brain acetaldehyde after ethanol administration. Biochem Pharmacol 25:1305-1309.

Taiwo YO, Levine JD (1989) Contribution of guanine nucleotide regulatory proteins to prostaglandin hyperalgesia in the rat. Brain Res 492:400-403.

Taiwo YO, Levine JD (1991) Further confirmation of the role of adenyl cyclase and of cAMP-dependent protein kinase in primary afferent hyperalgesia. Neuroscience 44:131-135.

Taiwo YO, Bjerknes LK, Goetzl EJ, Levine JD (1989a) Mediation of primary afferent peripheral hyperalgesia by the cAMP second messenger system. Neuroscience 32:577-580.

Taiwo YO, Coderre TJ, Levine JD (1989b) The contribution of training to sensitivity in the nociceptive paw-withdrawal test. Brain Res 487:148-151. 
Tanner KD, Reichling DB, Levine JD (1998) Nociceptor hyperresponsiveness during vincristine-induced painf ul peripheral neuropathy in the rat. J Neurosci 18:6480-6491.

Tredici G, Miloso M, Nicolini G, Galbiati S, Cavaletti G, Bertelli A (1999) Resveratrol, MAP kinases and neuronal cells: might wine be a neuroprotectant? Drugs Exp Clin Res 25:99-103.

Tsapis A, Kepes A (1977) Transient breakdown of the permeability barrier of the membrane of Escherichia coli upon hypoosmotic shock. Biochim Biophys Acta 469:1-12.

Weise F, Muller D, Krell D, Kielstein V, Koch RD (1985) Autonomic alcoholic neuropathy. Z Gesamte Inn Med 40:160-162.

West LK, Huang L (1980) Transient permeabilization induced osmotically in membrane vesicles from Torpedo electroplax: a mild procedure for trapping small molecules. Biochemistry 19:4418-4423.

Widdicombe JH, Azizi F, Kang T, Pittet JF (1996) Transient permeabilization of airway epithelium by mucosal water. J Appl Physiol 81:491-499.
Willis Jr WD (1985) The pain system. The neural basis of nociceptive transmission in the mammalian nervous system. Pain Headache 8:1-346.

Xie YK, Xiao WH (1990) Electrophysiological evidence for hyperalgesia in the peripheral neuropathy. Sci China B 33:663-672.

Yokoyama A, Takagi T, Ishii H, Muramatsu T, Akai J, Kato S, Hori S, Maruyama K, Kono H, Tsuchiya M (1991) Impaired autonomic nervous system in alcoholics assessed by heart rate variation. Alcohol Clin Exp Res 15:761-765.

Yoon YW, Na HS, Chung JM (1996) Contributions of injured and intact afferents to neuropathic pain in an experimental rat model. Pain 64:27-36.

Yoshimura M, Tabakoff B (1999) Ethanol's actions on cAMP-mediated signaling in cells transfected with type VII adenylyl cyclase. Alcohol Clin Exp Res 23:1457-1461.

Zahn PK, Brennan TJ (1999) Primary and secondary hyperalgesia in a rat model for human postoperative pain. Anesthesiology 90:863-872. 